\title{
Population health status based on the EQ-5D-Y-3L among adolescents in Sweden: Results by sociodemographic factors and self-reported comorbidity
}

\author{
Mimmi Åström ${ }^{1,2,3}$ (1) Carina Persson ${ }^{4,5} \cdot$ Margareta Lindén-Boström $^{4,5} \cdot$ Ola Rolfson $^{1,6,7} \cdot$ Kristina Burström $^{1,2,3}$
}

Accepted: 28 August 2018 / Published online: 8 September 2018

(c) The Author(s) 2018

\begin{abstract}
Purpose The EQ-5D-Y-3L is a generic health-related quality of life (HRQoL) measure developed for youth from 8 years old. The aim of this study is to present population health status, based on the EQ-5D-Y-3L, among adolescents in Sweden, by sex, age, self-reported comorbidity and parents' occupational status.

Methods Data were obtained from a cross-sectional total survey among students, aged 13-18 years, in a Swedish County year 2014. The survey included EQ-5D-Y-3L, questions regarding self-reported health, disease, functional impairment and mental distress. Parents' occupational status was used as a proxy for socio-economic status.

Results A total of 6574 participants answered all the EQ-5D-Y-3L dimensions (mean age was 15.9 years, same proportion of boys and girls). Girls reported more problems than boys in the dimensions 'doing usual activities', 'having pain or discomfort' and 'feeling worried, sad or unhappy', and lower mean VAS score. Respondents with one or both parents unemployed reported more problems with usual activities, pain/discomfort and in the mood dimension than those with both parents employed. Those with comorbidity had in general more problems in all dimensions and lower mean VAS score. The highest impact on VAS score was found for adolescents who reported that they always felt depressed.

Conclusions Sex, age, self-reported comorbidity and parents' occupational status were associated with HRQoL determined by the EQ-5D-Y-3L in the general population of adolescents. The ability of EQ-5D-Y-3L to distinguish adolescents' health status based on these factors confirms the instrument's usefulness in assessment of HRQoL and as guidance for prioritization.
\end{abstract}

Keywords Adolescents · EQ-5D-Y-3L · Functional impairment · General population · Mental distress · Parents' occupational status

Electronic supplementary material The online version of this article (https://doi.org/10.1007/s11136-018-1985-2) contains supplementary material, which is available to authorized users.

Mimmi Åström

mimmi.astrom@ki.se

1 Health Outcomes and Economic Evaluation Research Group, Stockholm Centre for Healthcare Ethics, Department of Learning, Informatics, Management and Ethics, Karolinska Institutet, Tomtebodavägen $18 \mathrm{~A}$, 17177 Stockholm, Sweden

2 Equity and Health Policy Research Group, Department of Public Health Sciences, Karolinska Institutet, Stockholm, Sweden

3 Health Care Services, Stockholm County Council, Stockholm, Sweden
4 Department for Sustainable Development, Region Örebro County, Örebro, Sweden

5 Faculty of Medicine and Health, Örebro University, Örebro, Sweden

6 Department of Orthopaedics, Institute of Clinical Sciences, the Sahlgrenska Academy, University of Gothenburg, Gothenburg, Sweden

7 Swedish Hip Arthroplasty Register, Gothenburg, Sweden 


\section{Introduction}

Each child has the right to the highest achievable health as well as to access to health care, treatment and rehabilitation [1]. Measuring health status in all ages is important when describing and monitoring health in a population and when evaluating treatment effects and conducting economic evaluation in health care [2-4].

Many health care systems emphasize that health should be measured from the perspective of the patient as a complement to clinical measures [5]. This can be achieved by the use of patient-reported outcome measures (PROMs), which are standardized instruments established to capture the patient's health from the patient's own point of view $[5,6]$. Several PROMs, which include measure of healthrelated quality of life (HRQoL), targeting children and adolescents have been developed [2, 4, 7]. HRQoL is a multidimensional description of health and includes physical, psychological, social and emotional dimensions that are influenced by changes in health status and important for a person's overall well-being [8]. HRQoL can be measured with generic or condition-specific instruments [3].

Using self-reported PROMs in children and adolescents entails several methodological challenges, like the child's development stage, general cognitive competence and understanding of the concepts of health and illness [7, 9, 10]. Proxy measures, where e.g. parents or health care personnel are asked to report the child's health on behalf of the child have been used as an alternative to children self-reporting their own health [4, 7]. However, since the concept of HRQoL refers to the individual's own perception of health status, it is desirable to derive information directly from the individual of interest $[10,11]$. It has been observed that children from the age of eight can self-report their health in a meaningful way and for children from 12 years old, self-report is preferred [9, 12]. Nevertheless, each child's pace of development is unique and a clear age cut-off from when children are able to self-report their health is not possible to state [12].

Collecting HRQoL data from the general population of children and adolescents makes it possible to monitor population health status over time and identify groups within the general population with greater risk of poor health [13]. Furthermore, it enables comparisons of health status of the general population with specific patient groups [13-15]. Population data describe health among the general population, commonly by sex, age and socioeconomic status. Population data, also referred to as population norms or population reference data [13], are usually collected through general population health surveys [16]. The adult version of the EQ-5D has been used to measure health status in many countries and regions and population data have been established based on the instrument [13, 17, 18]. There are health surveys targeting children and adolescents, but those usually do not include self-reported HRQoL instruments.

The EQ-5D-Y-3L is a generic HRQoL instrument developed by modifying the language and layout of the adult version EQ-5D-3L to make the instrument suitable for children from 8 years old $[19,20]$. The EQ-5D-Y-3L is translated to over 40 languages and offers a range of modes of administration, such as self-completion paper and pencil version and versions for tablets and smartphones [21]. The EQ-5D-Y-3L has been tested in terms of feasibility, validity and reliability in general populations of children and adolescents [22, 23]. In clinical studies, the EQ-5D-Y-3L has been validated for a number of health conditions among children and adolescents, such as cystic fibrosis, functional disabilities, asthma and among acutely ill children [24-27]. Population health status among children aged between 7 and 12 years old has been investigated with the EQ-5D-Y-3L instrument [23, 28, 29].

To the best of our knowledge, no population data exist for the general population of adolescents based on the EQ5D-Y-3L instrument. The aim of this study is to present population health status, based on the EQ-5D-Y-3L, among adolescents in Sweden, by sex, age, self-reported comorbidity and parents' occupational status.

\section{Methods}

\section{Study design}

Data were obtained from the general population survey Life \& Health-young people conducted in year 2014 among Swedish adolescents in Örebro County. The self-administered paper and pencil survey was distributed to all adolescents in grade seven and nine in compulsory school, and in the second year of upper secondary school. The purpose of the survey was to investigate adolescents living conditions, health-related behaviours and health, results from the survey are described elsewhere [30]. The survey was distributed in two versions, one to adolescents in grade seven and one to adolescents in grade nine and in the second year of upper secondary school. Whereas a majority of questions were common between surveys, questions regarding e.g. sexual behaviours and illicit drugs were only asked to adolescents in school year nine and in the second year of upper secondary school.

Teachers or principals at each school informed the pupils about the survey and invited them to answer the survey anonymously in the classroom during school hours. Teachers informed that participation was voluntary that they could withdraw from participation, and that collected data could 
not be traced to the individual. In the survey, there was written information regarding participation. After completion, participants were asked to put the survey in an envelope and seal it. Prior to the data collection, parents/guardians were informed about the purpose of the survey that participation was voluntary and that they could withdraw their adolescent from participation. Ethical approval was granted by the Regional Ethical Review Board in Uppsala, Sweden (Dnr: 2013/459).

\section{Measures}

The survey consisted of 62 questions for adolescents in school year seven, and 86 questions for adolescents in school year nine and in the second year of upper secondary school. The survey covered questions regarding health, healthrelated behaviours and living conditions, e.g. questions on socio-demographics, self-rated health (SRH), parents' occupational status, self-reported disease and functional impairment. In year 2014, the survey included, for the first time, the Swedish version of the EQ-5D-Y-3L instrument.

\section{EQ-5D-Y-3L}

The EQ-5D-Y-3L consists of a descriptive system with five dimensions and a visual analogue scale (VAS), where the respondent is asked to self-report his or her health status today $[19,20]$. EQ-5D-Y-3L consists of the following dimensions: 'mobility', 'looking after myself', 'doing usual activities', 'having pain or discomfort' and 'feeling worried, sad or unhappy'. Each dimension has three severity levels: 'no' problems, 'some' problems and 'a lot of' problems. A total of $243\left(3^{5}\right)$ unique health profiles can be derived from the dimensions in combination with the severity levels. For the VAS, the respondent is asked to rate their overall health status between 100 (the best health you can imagine) and 0 (the worst health you can imagine) [19].

\section{Self-rated health}

SRH is a single question frequently used in surveys to assess respondent's self-reported health [31, 32]. The SRH question was phrased 'How is your overall health?' The response options were as follows: 'very good, good, neither good nor bad, bad, very bad'.

\section{Parents' occupational status}

In the absence of information on the socio-economic status of parents, parents' occupational status was used as a proxy. Parents' occupational status was assessed by a multiple-choice question regarding the respondent's father's and mother's occupational status. Socio-economic status based on adolescents' reports of parents' occupational status has been used earlier [33]. In this survey, the questions were framed 'What does your father and mother do?' The response options were as follows: 'working, on sick leave/ disability pension, unemployed, studying, on parental leave, other, do not know'. Respondents who chose more than one response option or who did not answer the questions were excluded ( $n=1520)$. Father's and mother's occupational status were combined into parents' occupational status (both parents working vs. one or both parents being unemployed).

\section{Self-reported disease}

Self-reported disease was assessed by the multiple-choice question 'Do you have any of the following diseases? Asthma, allergic eyes or nasal symptoms, food allergy, nickel allergy, eczema, other skin disease, diabetes, or/and epilepsy?' The answers were dichotomized into yes (mild and severe disease) and no (not having the disease) in the analysis.

\section{Self-reported functional impairment}

Self-reported functional impairment was assessed by the multiple-choice question 'Do you have any of the following functional impairments? Hearing loss, vision loss, physical impairment, reading- and writing disabilities or dyslexia, Attention Deficit Hyperactivity Disorder (ADHD) (Asperger, Tourette or similar) or/and other functional impairment?' The answers were dichotomized into yes (mild and severe functional impairment) and no (not having the functional impairment) in the analysis.

\section{Body mass index}

Body mass index (BMI) was calculated from self-reported height and weight of the individual. BMI was calculated by dividing body weight in kilograms by height in meters squared $\left(\mathrm{kg} / \mathrm{m}^{2}\right)$ for each respondent. Cut-off points were for underweight $<18.5$, normal weight $\geq 18.5<25$, overweight $\geq 25<30$ and obesity $\geq 30$ [34].

\section{Mental distress}

Mental distress was assessed by two questions 'During the past 3 months, how often have you felt stressed?' and 'During the past 3 months, how often have you felt depressed?' The response options were as follows: 'never, rarely, sometimes, often, always'. The answers were dichotomized into yes (always or often) and no (never, rarely or sometimes) in the descriptive analysis. 


\section{Data analyses}

Inclusion criterion was being aged 13-18 years at the end of year 2014 i.e. born between the year 1996 and 2001 . Respondents who did not provide an answer about sex or age were excluded. Regarding the EQ-5D-Y-3L, a complete case analysis was chosen; hence, respondents with missing values on any of the dimensions were deleted. The complete cases analysis is recommended when the proportion of missing values is small, around $<5 \%$ [35].

The sample was divided into three age groups: 13-14 years, 15-16 years and 17-18 years. Calculation of the proportion of adolescents reporting 'no', 'some' and 'a lot of' problems in each EQ-5D-Y-3L dimension was assessed by sex, age group, parents' occupational status, disease, functional impairment, mental distress, SRH and BMI. To test for statistical significant differences between groups in proportion of reported problems, the Chi-square test or the Fisher's Exact test was used. Prior to the significance test, the severity levels 'some' problems and 'a lot of' problems were combined into 'any' problems. The Mann-Whitney $U$ test was used to test for statistical significant differences in mean VAS scores between groups [36]. Multiple logistic regression analysis was used to investigate associations between reported problems in the EQ-5D-Y-3L dimensions and respondent's sex, age and parent's occupational status. The results are presented as odds ratio (OR). Multiple linear regression was used to investigate the association between mean VAS score and sex, age group, parents' occupational status, disease, functional impairment, mental distress and BMI. A 5\% significance level was used and analyses were performed in SPSS 23 [37].

\section{Results}

\section{Response rate}

In total, 7399 pupils answered the survey and the response rate was $79.7 \%$. Respondents with missing or ambiguous answers for sex $(1.6 \%)$, age $(0.9 \%)$ and not fulfilling the inclusion criteria of age were excluded, which resulted in 6805 respondents. Those with missing answers on one or more $(n=231,3.4 \%)$ of the EQ-5D-Y-3L dimensions ('mobility' $0.9 \%$; 'looking after myself' $1.4 \%$; 'doing usual activities' $1.4 \%$; 'having pain or discomfort' $1.7 \%$; 'feeling worried, sad or unhappy' $1.8 \%$ ) were excluded. The final sample for analysis consists of 6574 participants. In the final sample, $106(1.6 \%)$ participants had a missing VAS score.

\section{Characteristics of study participants}

The mean age was 15.9 years and boys comprised half of the sample. A majority of the participants reported that both their parents were working $(73.1 \%)$. The most commonly self-reported disease and functional impairment were allergic eyes or nasal symptoms $(21.5 \%)$, and reading- and writing disabilities or dyslexia (7.3\%). Among the respondents, $31.8 \%$ reported often or always felt stressed, and $14.0 \%$ reported often or always felt depressed, during the past 3 months (Table 1).

\section{Health profiles}

There were in total 94 unique health profiles in the sample. Most frequently reported (44.9\%) was the health profile 11111 (i.e. no problems in any of the EQ-5D-Y-3L dimensions). The health profile 33333 (i.e. a lot of problems in all dimensions) was reported by two respondents (Online Appendix Table 1).

\section{EQ-5D-Y-3L by sex and age group}

In total, most problems were reported in the dimensions 'having pain or discomfort' and 'feeling worried, sad or unhappy'. Differences in proportion of reported problems between boys and girls were found, girls reported more problems in the dimensions 'doing usual activities', 'having pain or discomfort' and 'feeling worried, sad or unhappy'. Highest proportion of reported problems for girls was in the mood dimension and for boys with pain and discomfort. Girls reported lower mean VAS score (71.8) than did boys (78.9) (Table 2).

The youngest age group, 13-14 years, were those who reported most problems in the 'mobility' dimension. Respondents aged 15-16 years reported most problems in the dimensions 'doing usual activities', 'having pain or discomfort' and 'feeling worried, sad or unhappy', compared to the other age groups. The youngest age group, 13-14 years, reported the highest mean VAS score (78.0), compared to the older age groups (Table 2).

Girls in all age groups reported most problems in the mood dimension while boys, in all age groups, reported most problems with pain/discomfort. In the youngest age group, 13-14 years, girls reported more problems than boys did with pain/discomfort and in the mood dimension. In the age groups 15-16 years and 17-18 years, girls reported more problems than boys in the dimensions 'doing usual activities', 'having pain or discomfort' and 'feeling worried, sad or unhappy'. Girls in all age groups reported lower mean VAS scores than boys, in particular in the age group 15-16 years (69.6) (Table 2). 
Table 1 Characteristics of study participants $(n=6574)$

\begin{tabular}{|c|c|c|}
\hline & $\%$ & $n$ \\
\hline Age years mean (SD) & 15.9 & (1.6) \\
\hline \multicolumn{3}{|l|}{ Age groups (years) } \\
\hline $13-14$ & 34.3 & 2252 \\
\hline $15-16$ & 34.3 & 2254 \\
\hline $17-18$ & 31.5 & 2068 \\
\hline \multicolumn{3}{|l|}{ Sex } \\
\hline Boys & 50.6 & 3324 \\
\hline Girls & 49.4 & 3250 \\
\hline \multicolumn{3}{|l|}{ Socio-economic status } \\
\hline Both parents work & 73.1 & 4805 \\
\hline One or both parents unemployed & 3.8 & 249 \\
\hline Missing & 23.1 & 1520 \\
\hline \multicolumn{3}{|l|}{ Self-rated health } \\
\hline Very good & 39.6 & 2601 \\
\hline Good & 43.7 & 2870 \\
\hline Neither good or bad & 11.9 & 785 \\
\hline $\mathrm{Bad}$ & 3.1 & 203 \\
\hline Very bad & 1.1 & 70 \\
\hline Missing & 0.7 & 45 \\
\hline \multicolumn{3}{|l|}{ Self-reported disease } \\
\hline Allergic eyes/nasal symptoms & 21.5 & 1415 \\
\hline Eczema & 11.8 & 775 \\
\hline Asthma & 11.4 & 749 \\
\hline Nickel allergy & 9.0 & 592 \\
\hline Food allergy & 7.2 & 475 \\
\hline Other skin disease & 3.9 & 257 \\
\hline Diabetes & 0.8 & 52 \\
\hline Epilepsy & 0.6 & 38 \\
\hline \multicolumn{3}{|l|}{ Self-reported functional impairment } \\
\hline Reading- and writing disabilities or dyslexia & 7.3 & 481 \\
\hline Hearing loss & 6.2 & 409 \\
\hline ADHD, Asperger, Tourette or similar & 4.5 & 299 \\
\hline Vision loss & 3.8 & 251 \\
\hline Physical disability & 2.3 & 150 \\
\hline Other functional disability & 2.2 & 142 \\
\hline \multicolumn{3}{|l|}{ Self-reported BMI } \\
\hline Underweight & 8.4 & 555 \\
\hline Normal weight & 66.4 & 4366 \\
\hline Overweight & 12.4 & 818 \\
\hline Obesity & 3.0 & 195 \\
\hline Missing & 9.7 & 640 \\
\hline \multicolumn{3}{|l|}{ Self-reported stress ${ }^{\mathrm{a}}$} \\
\hline Never stressed & 10.2 & 673 \\
\hline Rarely stressed & 23.6 & 1552 \\
\hline Sometimes stressed & 33.7 & 2217 \\
\hline Often stressed & 26.2 & 1720 \\
\hline Always stressed & 5.6 & 370 \\
\hline Missing & 0.6 & 42 \\
\hline
\end{tabular}

Table 1 (continued)

\begin{tabular}{lrr}
\hline & $\%$ & $n$ \\
\hline Self-reported depression $^{\mathrm{a}}$ & & \\
Never depressed & 26.9 & 1771 \\
Rarely depressed & 32.3 & 2125 \\
Sometimes depressed & 24.3 & 1595 \\
Often depressed & 12.0 & 787 \\
Always depressed & 2.0 & 132 \\
Missing & 2.5 & 164 \\
\hline
\end{tabular}

${ }^{\text {a}}$ During the past 3 months

\section{EQ-5D-Y-3L by parents' occupational status}

Respondents with one or both parents being unemployed compared to those with both parents working, reported more problems with usual activities, pain/discomfort and in the mood dimension. Respondents with one or both parents being unemployed reported lower mean VAS score (Table 3).

\section{EQ-5D-Y-3L by self-reported disease, functional impairment and mental distress}

Respondents with asthma, allergic eyes/nasal symptoms, food allergy, nickel allergy and skin disease reported more problems in all EQ-5D-Y-3L dimensions except in the dimension 'looking after myself', and lower mean VAS score, than those not reporting that specific disease. No differences in reported problems were found for respondents with diabetes compared to those not reporting diabetes. Respondents with epilepsy had most problems across all dimensions, except for the mood dimension, and had the lowest mean VAS score (65.4) (Table 4).

Respondents with impairment reported more problems across all EQ-5D-Y-3L dimensions and lower mean VAS score than those not reporting that specific impairment. Among respondents with a physical impairment, $82.0 \%$ had problems with pain/discomfort; further, this group reported most problems across all dimensions, except the mood dimension, and the lowest mean VAS score (62.0) (Table 4).

Respondents, who often or always felt stressed or depressed during the past 3 months, reported more problems in all EQ-5D-Y-3L dimensions except in the dimensions 'looking after myself', and lower VAS score compared to those reported mental distress never, rarely or sometimes. Among those reporting always felt depressed, 90.7\% had problems in the mood dimension (Table 4). 


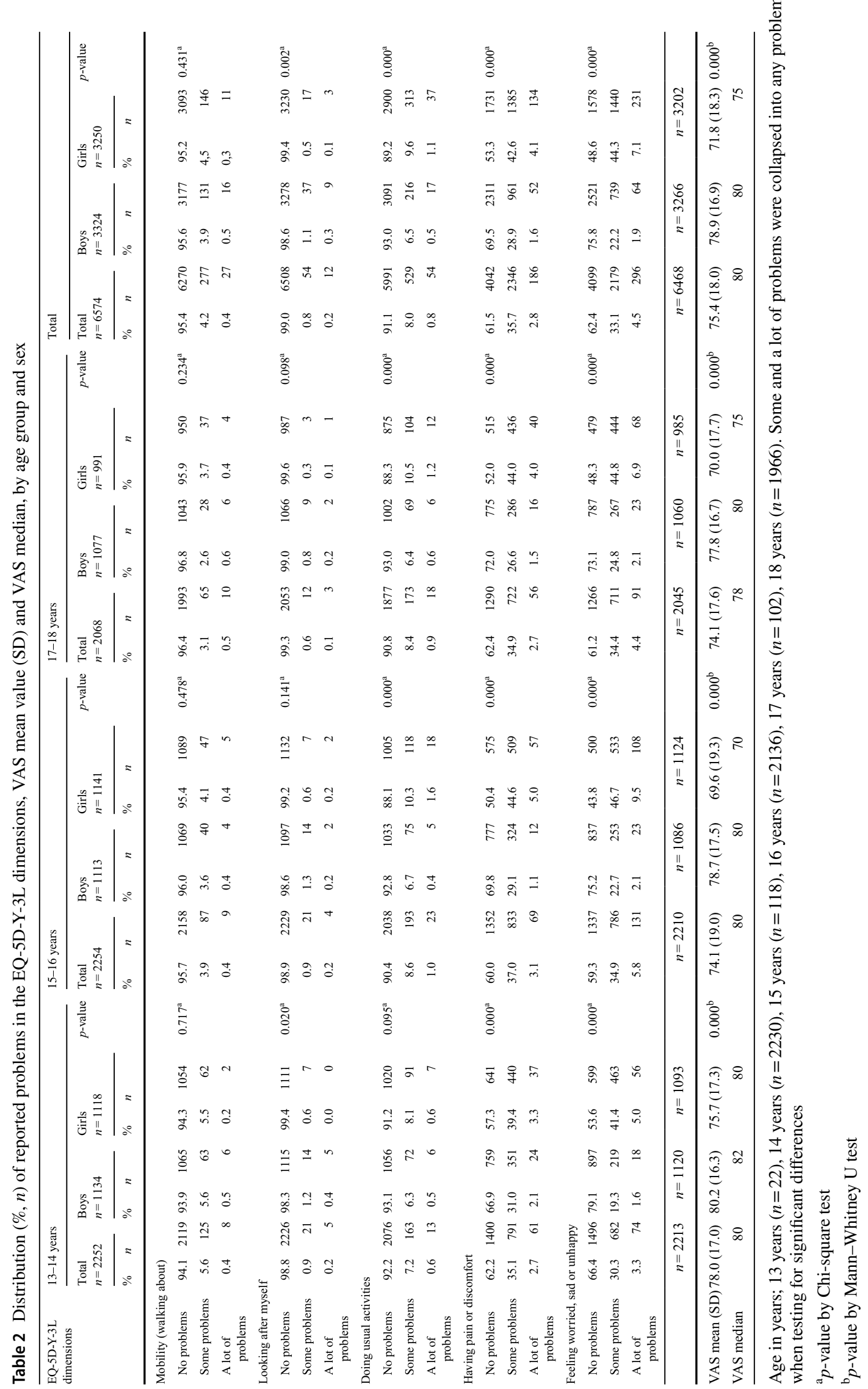


Table 3 Distribution $(\%, n)$ of reported problems in the EQ-5D-Y-3L dimensions, VAS mean value (SD) and VAS median by parents' occupational status

\begin{tabular}{|c|c|c|c|c|c|}
\hline \multirow[t]{3}{*}{ EQ-5D-Y-3L dimensions } & \multirow{2}{*}{\multicolumn{2}{|c|}{$\begin{array}{l}\text { One or both parents } \\
\text { unemployed }\end{array}$}} & \multicolumn{2}{|c|}{ Both parents work } & \multirow[t]{3}{*}{$p$-value } \\
\hline & & & \multicolumn{2}{|c|}{$n=4805$} & \\
\hline & $\%$ & $n$ & $\%$ & $n$ & \\
\hline \multicolumn{6}{|l|}{ Mobility (walking about) } \\
\hline Some problems & 3.2 & 8 & 3.6 & 175 & \multirow[t]{2}{*}{$0.527^{\mathrm{a}}$} \\
\hline A lot of problems & 0.0 & 0 & 0.4 & 18 & \\
\hline \multicolumn{6}{|l|}{ Looking after myself } \\
\hline Some problems & 1.6 & 4 & 0.7 & 35 & \multirow[t]{2}{*}{$0.286^{\mathrm{b}}$} \\
\hline A lot of problems & 0.0 & 0 & 0.1 & 7 & \\
\hline \multicolumn{6}{|l|}{ Doing usual activities } \\
\hline Some problems & 10.0 & 25 & 7.1 & 343 & \multirow[t]{2}{*}{$0.030^{\mathrm{a}}$} \\
\hline A lot of problems & 1.6 & 4 & 0.7 & 33 & \\
\hline \multicolumn{6}{|l|}{ Having pain or discomfort } \\
\hline Some problems & 44.2 & 110 & 34.8 & 1670 & \multirow[t]{2}{*}{$0.001^{\mathrm{a}}$} \\
\hline A lot of problems & 3.2 & 8 & 2.5 & 121 & \\
\hline \multicolumn{6}{|c|}{ Feeling worried, sad or unhappy } \\
\hline Some problems & 40.6 & 101 & 31.4 & 1,51 & \multirow[t]{2}{*}{$0.000^{\mathrm{a}}$} \\
\hline \multirow[t]{2}{*}{ A lot of problems } & 6.4 & 16 & 3.8 & 181 & \\
\hline & \multicolumn{2}{|c|}{$n=244$} & \multicolumn{3}{|c|}{$n=4737$} \\
\hline VAS mean (SD) & \multicolumn{2}{|c|}{$72.3(18.8)$} & \multicolumn{2}{|r|}{$76.1(17.3)$} & \multirow[t]{2}{*}{$0.000^{\mathrm{c}}$} \\
\hline VAS median & & 75 & & 80 & \\
\hline
\end{tabular}

Some and a lot of problems were collapsed into any problems when testing for significant differences ${ }^{\text {a }} p$-value by Chi-square test

${ }^{\mathrm{b}} p$-value by Fisher's Exact Test

${ }^{c} p$-value by Mann-Whitney U test

\section{EQ-5D-Y-3L by self-rated health}

There was a gradient in reported problems in the EQ-5D-Y3L dimensions from 'very good' to 'very bad' SRH (Online Appendix Fig. 1). Respondents who answered 'very bad' to the SRH question reported the lowest mean VAS score (Online Appendix Fig. 2).

\section{EQ-5D-Y-3L by body mass index}

Adolescents who had a $\mathrm{BMI} \geq 30$ and thus classified as obese, reported most problems across all EQ-5D-Y-3L dimensions. Among respondents classified as obese, the highest proportion of reported problems were with pain/ discomfort and in the mood dimension. Respondents classified as obese also reported the lowest mean VAS score (66.9) (Online Appendix Table 2).

\section{Regression analyses}

Controlling for age and parents' occupational status, girls were more likely than boys to report 'some' or 'a lot' problems in the dimensions 'doing usual activities', 'having pain or discomfort' and 'feeling worried, sad or unhappy' (Table 5). For girls compared to boys, the highest odds (OR 3.44) of reporting any problems was found for the mood dimension. Regarding age, adolescents in the age group 15-16 years were more likely to have problems in the mood dimension and less likely to report problems in the 'mobility' dimension, compared to the youngest age group. Controlling for age and sex, adolescents with one or both parents being unemployed were more likely to report problems with usual activities, pain/discomfort and in the mood dimension (Table 5).

Variation of VAS score by sex, age, self-reported disease, functional impairment, mental distress, parents' occupational status and BMI is shown in Table 6. Girls reported lower VAS score than boys and the older age groups (15-16 years and 17-18 years) reported lower VAS score than those 13-14 years old (Model 1). Controlling for sex and age, adolescents with one or both parents being unemployed reported lower VAS score (Model 2). After controlling for sex and age, adolescents with underweight, overweight and obesity, had lower VAS score compared to those with normal weight (Model 3). These differences remained when also controlling for parents' occupational 


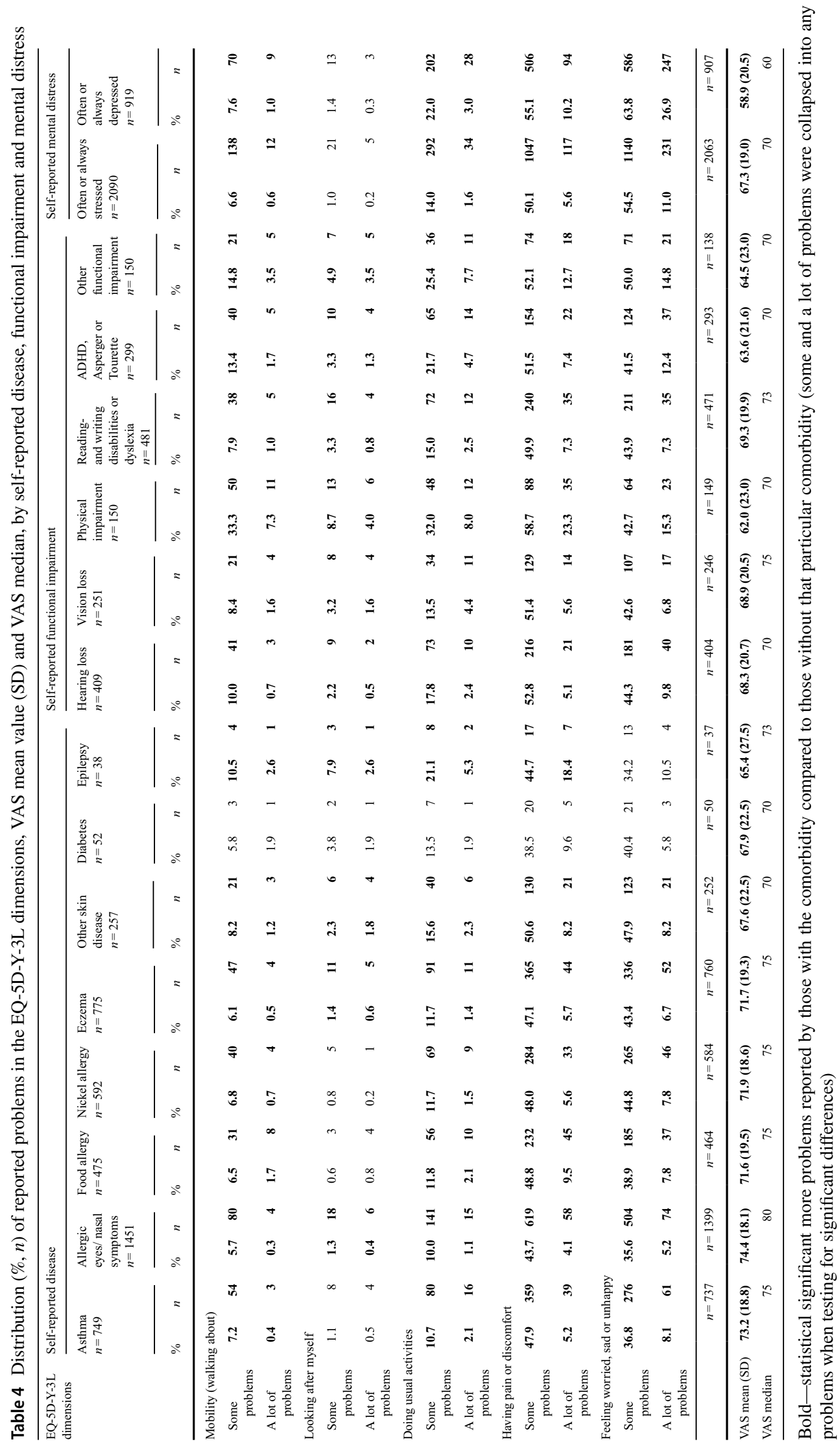


status (Model 4). After controlling for sex and age, adolescents who self-reported other skin disease, diabetes, epilepsy, reading or writing disabilities or dyslexia, hearing loss, ADHD, physical impairment, stress or depression, reported lower VAS score compared to those not having the disease or the functional impairment, or never feeling stressed or depressed (Model 5). When also controlling for parents' occupational status, the association with VAS score remained for all diseases, functional impairments and mental distress, except for respondents with diabetes (Model 6). The highest association with VAS score was found for adolescents who reported feeling depressed "always" for the past 3 months (28.6), controlling for all other factors (Model 7).

\section{Discussion}

This is the first study presenting population health status, based on the EQ-5D-Y-3L, among adolescents in Sweden, by sex, age, self-reported comorbidity and parents' occupational status. The study shows how adolescents in a general population report their subjective HRQoL and how factors such as disease, functional impairment, and mental distress associate with HRQoL. The study identifies girls compared to boys, older age groups compared to younger and those with one or both parents being unemployed, having reduced HRQoL, measured with the EQ-5D-Y-3L.

The fact that girls reported worse health status than did boys has not been found in earlier studies using the EQ5D-Y-3L [23, 25, 26]. Although, in studies among adults, using the EQ-5D-3L, women commonly report worse health status than men $[14,17]$. In the present study, the observed differences in reported problems in the dimensions and in VAS score between boys and girls, remained significant even after controlling for other factors. The high prevalence of reported problems among girls in the mood dimension is alarming and a cause for concern. Especially noticeable in the age group 15-16 years, where nearly $10 \%$ reported 'a lot of' problems in the mood dimension. A similar finding, with a relatively high prevalence of reported problems in the mood dimension, has been seen among young women in Sweden [17]. This is also in line with previous results among adolescents, where girls have been reporting worse subjective health than boys [38].

Despite that the present study was conducted among a general population of adolescents, more than half of all respondents reported problems in at least one of the EQ5D-Y-3L dimensions which was higher than observed earlier [29]. The age range of participants in our study could explain these findings, as it is recognized that adolescents go through several life-challenging changes e.g. increased social pressure from peers and detaching from parents, which can affect HRQoL [39]. Differences in both directions, regarding 


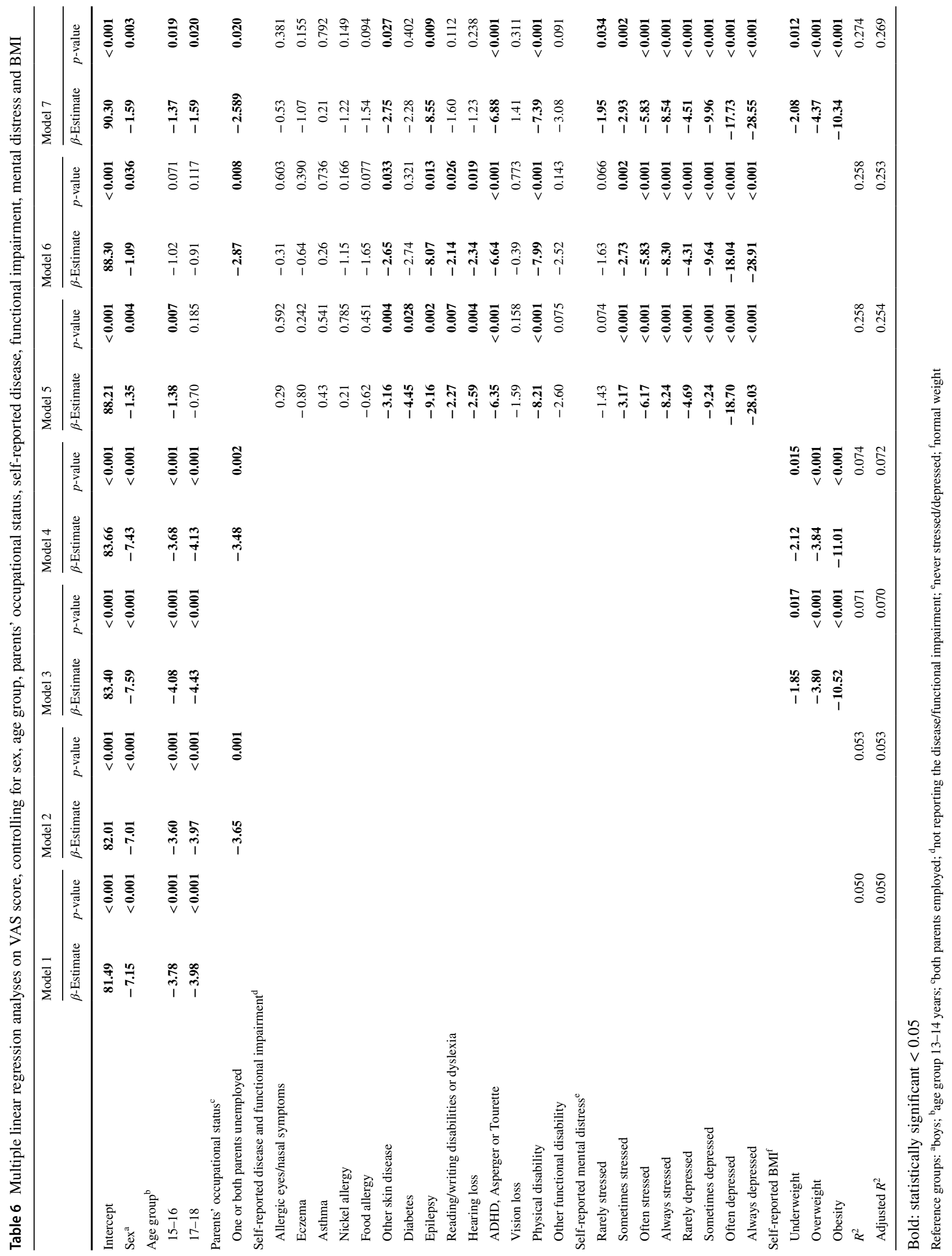


HRQoL measured with the EQ-5D-Y-3L instrument between younger ages have been observed $[23,26]$ but more studies are needed to investigate differences in HRQoL between early and late adolescence. In line with the findings from the present study, adolescents have reported lower HRQoL than children, using another instrument [40]. It is of importance to stress potential differences in HRQoL between late and early adolescence, when comparing health of specific patient groups with general population data.

The positive association between health and socio-economic status is well known [41]. Children's health status have shown to be positively associated with household income and the association even more distinct as children grow older into adolescence [42]. How to measure socioeconomic status among children and adolescents has been discussed, previously parents' occupation, income level and educational level have been used as indicators [28, 29]. In the present study, parents' occupational status reported by the adolescent was used as a proxy for socio-economic status. Adolescents with one or both parents unemployed reported worse health status. This is in line with previous studies, where children from families with the lowest household income showed the lowest VAS index scores, and children with parents with the lowest educational level reported more problems in the EQ-5D-Y-3L dimensions [28, 29]. If adolescents are able to report their parents' occupational status in a reliable matter can be discussed and might be a limitation of our study. However, good agreement between adolescents' reports and parents' self-reports regarding parents occupational status has been showed earlier [33].

Our study shows that self-reported disease, functional impairment and mental distress have a negative association with the prevalence of reported problems in the EQ-5D-Y-3L dimensions. Children and adolescents with health conditions or functional disability have also reported more problems in the dimensions and lower VAS score compared to those with no illness or functional disability [23]. Children and adolescents with a functional disability have also reported more problems compared to a general population sample [25]. Furthermore, in line with the findings from our study, children and adolescents with overweight or obesity have earlier reported more problems in all the dimensions and lower mean VAS score [23].

The overall results from the survey Life \& Health-young people are continuously used as a basis for decisions and priorities within the county, as well as for support for school health promotion [30]. To distribute the survey during school hours was successful as it resulted in a high response rate. This was the first year of including the EQ-5D-Y-3L instrument in the survey, and feasibility was indicated by few missing or ambiguous answers across all dimensions and for the VAS. Feasibility of the EQ-5D-Y-3L has been investigated in a similar way in previous studies [22, 23, 26, 29]. Two adolescents reported 'a lot of' problems in all the EQ-5D-Y$3 \mathrm{~L}$ dimensions. As the survey was distributed during school hours, it could be questioned whether adolescents with these amount of problems are able to attend school; however, these adolescents also answered very bad to the SRH question.

For the use of HRQoL instruments in economic assessments, there is a need to obtain a value attached to each health state to create a value set [43]. In the long term, it is essential to develop a value set for the EQ-5D-Y-3L to make it possible to use the instrument in economic evaluation of health care. Hence, as for today, there is no available value set for the EQ-5D-Y-3L [22], and it is not recommended to use the value set for the adult version for the EQ-5D-Y3L [44]. Studies, where adults have valued health states for children and adolescents based on the EQ-5D-Y-3L [45] and where adolescents have valued health states themselves based on other HRQoL measures [46], have been conducted. However, further studies to investigate how potential valuation methods works when used among children and adolescents are warranted.

\section{Conclusions}

The EQ-5D-Y-3L instrument is able to detect lower selfreported HRQoL among certain groups in the general population of adolescents. The results clearly show that sex, age and parents' occupational status are associated with health status. Furthermore, adolescents who self-report disease, functional impairment and mental distress report worse health status. When planning health interventions for adolescents, these findings can be used as guidance for prioritization, as all children and adolescents have the right to the highest achievable health.

Acknowledgements The authors would like to thank the adolescents for participating in the survey and Region Örebro County for providing microdata. Helpful comments on earlier versions of this paper when presented at the EuroQol Scientific Plenary Meeting 2016 and at the International Society for Pharmaeconomics and Outcomes Research (ISPOR) 2017 are thankfully acknowledged. Furthermore, valuable discussions in the Health Outcomes and Economic Evaluation Research Group and in the Equity and Health Policy Research Group at Karolinska Institutet are gratefully acknowledged. We appreciate fruitful suggestions from two anonymous reviewers.

Funding This study was funded by a research grant (Reference Number: 2015400) awarded by the EuroQol Research Foundation. The funder did not have any influence on the research process or the presentation of results.

\section{Compliance with ethical standards}

Conflict of interest Mimmi Åström and Kristina Burström are both members of the EuroQol Group. Carina Persson, Margareta LindénBoström, Ola Rolfson declare that they have no conflict of interest. 
Ethical approval All procedures performed in studies involving human participants were in accordance with the ethical standards of the institutional and/or national research committee and with the 1964 Helsinki Declaration and its later amendments or comparable ethical standards. This study reports results from an anonymous survey based on adolescents self-report. Ethical approval was granted by the Regional Ethical Review Board in Uppsala, Sweden (Dnr: 2013/459).

Informed consent Informed consent was obtained from all individual participants included in the study. In addition, parents and/or guardians were prior the data collection informed about the purpose of the survey that participation was voluntary and that they could withdraw their adolescent from participation.

Open Access This article is distributed under the terms of the Creative Commons Attribution 4.0 International License (http://creativeco mmons.org/licenses/by/4.0/), which permits unrestricted use, distribution, and reproduction in any medium, provided you give appropriate credit to the original author(s) and the source, provide a link to the Creative Commons license, and indicate if changes were made.

\section{References}

1. United Nations (UN). (1989). Convention on the rights of the child. Retrieved April 20, 2018, from http://www.ohchr.org/EN/ ProfessionalInterest/Pages/CRC.aspx.

2. Ravens-Sieberer, U., Erhart, M., Wille, N., Wetzel, R., Nickel, J., \& Bullinger, M. (2006). Generic health-related quality-of-life assessment in children and adolescents: Methodological considerations. Pharmacoeconomics, 24(12), 1199-1220.

3. Brazier, J., Ratcliffe, J., Salomon, J. A., \& Tsuchiya, A. (2007). Measuring and valuing health benefits for economic evaluation. New York: Oxford University Press.

4. Solans, M., Pane, S., Estrada, M. D., Serra-Sutton, V., Herdman, M., et al. (2008). Health-related quality of life measurement in children and adolescents: A systematic review of generic and disease-specific instruments. Value in Health, 11(4), 742-764.

5. Appleby, J., Devlin, N., \& Parkin, D. (2016). Using patient reported outcomes to improve health care. Chichester: Wiley.

6. Dawson, J., Doll, H., Fitzpatrick, R., Jenkinson, C., \& Carr, A. J. (2010). The routine use of patient reported outcome measures in healthcare settings. $B M J, 340, \mathrm{c} 186$.

7. Janssens, A., Thompson Coon, J., Rogers, M., Allen, K., Green, C., Jenkinson, C., et al. (2015). A systematic review of generic multidimensional patient-reported outcome measures for children, part I: Descriptive characteristics. Value in Health, 18(2), 315-333.

8. Kane, R. L., \& Radosevich, D. M. (2011). Conducting health outcomes research. Sudbury: Jones \& Bartlett Learning.

9. Riley, A. W. (2004). Evidence that school-age children can selfreport on their health. Ambulatory Pediatrics, 4(4), 371-376.

10. Ungar, W. J. (2010). Economic evaluation in child health. Oxford: Oxford University Press.

11. Noyes, J., \& Edwards, R. T. (2011). EQ-5D for the assessment of health-related quality of life and resource allocation in children: A systematic methodological review. Value in Health, 14(8), 1117-1129.

12. Matza, L. S., Patrick, D. L., Riley, A. W., Alexander, J. J., Rajmil, L., Pleil, A. M., et al. (2013). Pediatric patient-reported outcome instruments for research to support medical product labeling: Report of the ISPOR PRO good research practice for the assessment of children and adolescents task force. Value in Health, 16(4), 461-479.
13. Szende, A., Janssen, B., \& Cabases, J. (2014). Self-reported population health: An international perspective based on $E Q-5 D$. New York: Springer Open.

14. Sun, S., Irestig, R., Burström, B., Beijer, U., \& Burström, K. (2006). Health-related quality of life (EQ-5D) among homeless persons compared to a general population sample in Stockholm County, 2006. Scandinavian Journal of Public Health, 40(2), 115-125.

15. Ravens-Sieberer, U., Gosch, A., Abel, T., Auguier, P., Bellach, B. M., Bruil, J., et al. (2001). Quality of life in children and adolescents: A European public health perspective. Sozial and Praventivmedizin, 46(5), 294-302.

16. Ravens-Sieberer, U. (2002). Measuring and monitoring qualityof-life in population surveys: Still a challenge for public health research. Sozial and Praventivmedizin, 47(4), 203-204.

17. Burström, K., Johannesson, M., \& Diderichsen, F. (2001). Swedish population health-related quality of life results using the EQ-5D. Quality of Life Research, 10(7), 621-635.

18. Sorensen, J., Davidsen, M., Gudex, C., Pedersen, K. M., \& Bronnum-Hansen, H. (2009). Danish EQ-5D population norms. Scandinavian Journal of Public Health, 37(5), 467-474.

19. Wille, N., Badia, X., Bonsel, G., Burström, K., Cavrini, G., Devlin, N., et al. (2010). Development of the EQ-5D-Y: A child friendly version of the EQ-5D. Quality of Life Research, 19(6), $875-886$.

20. Burström, K., Egmar, A. C., Lugnér, A., Eriksson, M., \& Svartengren, M. (2010). A Swedish child-friendly pilot version of the EQ-5D instrument-The development process. European Journal of Public Health, 21(2), 171-177.

21. EuroQol Research Foundation. (2014). EQ-5D-Y user guideBasic information on how to use the EQ-5D-Y instrument. Retrieved April 20, 2018, from https://euroqol.org/wp-content/ uploads/2016/09/EQ-5D-Y_User_Guide_v1.0_2014.pdf.

22. Ravens-Sieberer, U., Wille, N., Badia, X., Bonsel, G., Burström, K., Cavrini, G., et al. (2010). Feasibility, reliability, and validity of the EQ-5D-Y: Results from a multinational study. Quality of Life Research, 19(6), 887-897.

23. Burström, K., Svartengren, M., \& Egmar, A.-C. (2010). Testing a Swedish child-friendly pilot version of the EQ-5D instrument-Initial results. European Journal of Public Health, 2(21), 178-183.

24. Eidt-Koch, D., Mittendorf, T., \& Greiner, W. (2008). Crosssectional validity of the EQ-5D-Y as a generic health outcome instrument in children and adolescents with cystic fibrosis in Germany. BMC Pediatric, 9, 55.

25. Burström, K., Bartonek, A., Brostrom, E. W., Sun, S., \& Egmar, A.-C. (2014). EQ-5D-Y as a health-related quality of life measure in children and adolescents with functional disability in Sweden: Testing feasibility and validity. Acta Paediatrica, 103(4), 426-435.

26. Bergfors, S., Åström, M., Burström, K., \& Egmar, A.-C. (2015). Measuring health-related quality of life with the EQ-5D-Y instrument in children and adolescents with asthma. Acta Paediatrica, 104, 167-173.

27. Scott, D., Ferguson, G. D., \& Jelsma, J. (2017). The use of the EQ-5D-Y health related quality of life outcome measure in children in the Western Cape, South Africa: Psychometric properties, feasibility and usefulness-A longitudinal, analytical study. Health and Quality of Life Outcomes, 15(1), 12.

28. Wu, X. Y., Ohinmaa, A., Johnson, J. A., \& Veugelers, P. J. (2014). Assessment of children's own health status using visual analogue scale and descriptive system of the EQ-5D-Y: Linkage between two systems. Quality of Life Research, 23(2), 393-402.

29. Kim, S. K., Jo, M. W., \& Kim, S. H. (2017). A cross sectional survey on health-related quality of life of elementary school students using the Korean version of the EQ-5D-Y. PeerJ, 5, e3115. 
30. Lindén-Boström, M., Löfwenhamn, E., \& Persson, C. (2015). Ungdomars livsvillkor, levnadsvanor och hälsa. Liv \& hälsa ung i Örebro län (Adolescents' living conditions, health-related behaviors and health. Life \& Health-Young people). Retrieved April 20, 2018, from https://www.regionorebrolan.se/Files-sv/\%c3\%96rebro\%20 $1 \%$ c3\%a4ns\%20landsting/V\%c3\%a5rd\%20och\%20h\%c3\%a4lsa/ Folkh\%c3\%a4lsa/Publikationer/Arkiv/2015_01_Ungdomars_livsv illkor_levnadsvanor_och_h\%c3\%a4lsa.pdf.

31. Mossey, J. M., \& Shapiro, E. (1982). Self-rated health: A predictor of mortality among the elderly. American Journal of Public Health, 72(8), 800-808.

32. Idler, E. L., \& Benyamini, Y. (1997). Self-rated health and mortality: A review of twenty seven community studies. Journal of Health and Social Behavior, 38(1), 21-37.

33. Lien, N., Friestad, C., \& Klepp, K.-I. (2001). Adolescents' proxy report of parents' socioeconomic status: How valid are they? Journal of Epidemiology and Community Health, 55, 731-737.

34. Cole, T. J., \& Lobstein, T. (2012). Extended international (IOTF) body mass index cut-offs for thinness, overweight and obesity. International Journal of Pediatric Obesity, 7(4), 284-294.

35. Fayers, P. M., \& Machin, D. (2007). Quality of life-The assessment, analysis and interpretation of patient-reported outcomes (2nd ed.). Chichester: Wiley.

36. Campbell, M. J., Machin, D., \& Walters, S. J. (2007). Medical statistics: A textbook for the health sciences (4th ed.). Chichester: Wiley.

37. IBM Corp. (2015). IBM SPSS statistics for windows. Version 23.0. Armonk, NY: IBM Corp.

38. Wiklund, M., Malmgren-Olsson, E. B., Öhman, A., Bergström, E., \& Fjellman-Wiklund, A. (2012). Subjective health complaints in older adolescents are related to perceived stress, anxiety and gender-A cross-sectional school study in Northern Sweden. BMC Public Health, 12, 993.

39. Koot, H. M., \& Wallander, J. L. (2001). Quality of life -In child and adolescent illness. East Sussex: Brunner-Routledge.

40. Bisegger, C., Cloetta, B., \& von Rueden, U. (2005). Healthrelated quality of life: Gender differences in childhood and adolescence. Sozial and Praventivmedizin, 50(5), 281-291.

41. Marmot, M., Ryff, C., Bumpass, L. L., Shipley, M., \& Marks, N. F. (1997). Social inequalities in health: Next questions and converging evidence. Social Science \& Medicine, 44(6), 901-910.

42. Case, A., Lubotsky, D., \& Paxson, C. (2002). Economic status and health in childhood. The origins of the gradient. The American Economic Review, 92(5), 1308-1334.

43. Drummond, M. F., Sculpher, M. J., Claxton, K., Stoddart, G. L., \& Torrance, G. W. (2015). Methods for the economic evaluation of health care programmes (4th ed.). New York: Oxford University Press.

44. Kind, P., Klose, K., Gusi, N., Olivares, P. R., \& Greiner, W. (2015). Can adult weights be used to value child health states? Testing the influence of perspective in valuing EQ-5D-Y. Quality of Life Research, 24(10), 19-39.

45. Craig, B. M., Greiner, W., Brown, D. S., \& Reeve, B. B. (2016). Valuation of child health-related quality of life in the United States. Health Economics, 25(6), 768-777.

46. Ratcliffe, J., Flynn, T., Terlich, F., Stevens, K., Brazier, J., \& Sawyer, M. (2012). Developing adolescent-specific health state values for economic evaluation: An application of profile case best-worst scaling to the Child Health Utility 9D. Pharmacoeconomics, 30(8), 713-727. 